\title{
Optimizing Human-Robot Integration in Block-Laying Task
}

\author{
B. Kahane and Y. Rosenfeld \\ Faculty of Civil Engineering and National Building Research Institute \\ Technion - Israel Institute of Technology \\ Haifa 32000, ISRAEL \\ Tc1/fax. 972-4-8292363 \\ E-mail: roseny@tx.technion.ac.il
}

\begin{abstract}
This paper presents a comparison of various ways to use a robotic system for building a wall. This task is analyzed in order to introduce a few optional systems that differ from each other by their level of autonomy and the task allocation between humans and the robot. An economic analysis is conducted in order to examine the profitability of each system. The economic implications are presented by tools that allow drawing specific conclusions adjusted to local circumstances.
\end{abstract}

\section{Introduction}

Constructing a building is composed of off-site and on-site work. In the long run, it is expected that the building components will be manufactured in fully automated factorics and then brought to the construction site, where they will be put together. On-site automated machines and robots will assemble most of the building and accomplish other tasks that could not have been done in the factories, mainly interior finishing tasks.

The use of off-site factories for manufacturing the pre-fabricated components of buildings is a well-known stage in industrialized construction. Automation and robotics have been developed during the years as a part of permanent factories and therefore they may be easily implemented in construction factories too. However, integrating robots in interior finishing works on-site is still innovative and dependent on much rescarch and development.

Previous research conducted at the Technion examined the appropriate configurations for interior finishing robots. Primer technical feasibility was examined in full-scale cxperiments, in which a multipurpose robot exccuted several interior finishing tasks (wall painting, tiling, partition building) [1]. Later research dealt with autonomous mapping of the work area [2] and optimization of the robot's moves on a floor [3].

A wide range of robotic systems for interior tasks was examined, mainly by research and development institutions around the world. A main parameter that distinguishes among them is their level of autonomy.
Various systems for brick or block laying were developed, bascd on different approaches. In order to achieve high degree of autonomy for the robot's work, special devices were required, such as sensiblc cnd-effectors [4] and complimentary devices $[5,6]$. Moreover, systems of high autonomy also need to transport themselves in a work area independently, hence their navigation and positioning abilities must also be developed, considering the site conditions and the task features. Some experimental results were obtained in using a laser beam and reflectors for a tumnel robot [7], and a laser beam relying on the existing columns was tested for an indoor marking robot [8]. Another research was aimed at comparing positioning methods for a brick-laying robot by simulating their performance [9].

- A different approach suggests using the robot as an assistant to the human worker, and accordingly the robot may be less autonomous, simpler in the technical sense, and using merely limited sensing abilities. Systems of less autonomous performance can bc more easily adapted to assist with a varicty of tasks. Basic systems of this kind are already in use, especially in Japan $[10,11,12]$.

The tendency of integrating robotic work in construction may lead to a vision in which a robot would be stationed at a construction sitc and picked up after accomplishing its task fully and autonomously. However, this vision seems to be utopian for the near future. In order to bridge the gap between the present and the distant future, it is necessary to scck intermediate steps that will advance gradually towards automated construction.

The aim of this paper is to present some variations of human-robot integration in executing interior finishing tasks. The process of building a partition with the robot's aid was examined in full scale. Several possible robotic systems are suggested for this task. Each one of the systems is analyzed, considering the requirements for executing the task completely, as a whole. The best output of every system was calculated, and the performance features are presented. An economic analysis was conducted in order to compare among the systems and draw some generalized conclusions. 
The results lead to a better understanding of possible man-robot intcgration, and highlight the relative profitability of future development trends.

\section{Building of partition walls}

The features of a partition-building task make its robotization very complicated. The position of the partition is absolute, so the construction method must be very accurate. At the same time, the task deals with erecting a new element, which is, therefore, difficult to identify or relate to.

There are three popular types of partition materials: concrete blocks, gypsum boards and interlocking gypsum blocks. The gypsum blocks (figure 1) were found to be the most appropriatc material for a robotized task due to their accurate sizes, their prime stability (as a result of their interlocking edges) and their smooth surface (that enables vacuum gripping).

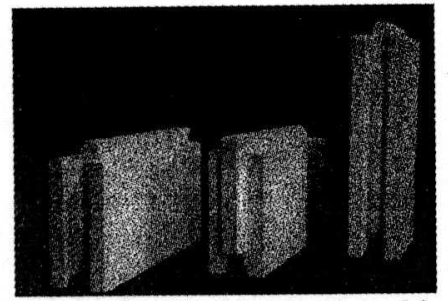

Figure 1: Interlocking gypsum blocks.

In the robotic construction method, the robot is placed in its position and builds the part of the wall in front of it (=working sector). Upon finishing the work at that sector, the robot moves to the next work station to build the remaining parts of the partition. The common assembling method is in stepped courses (figure 2), in which the progress is horizontal: the first row is to be built first, then the second row and so on. When a robot builds a sector, any row must be shorter than the one below. As a result, the average working sector has a parallelogram shape (figure 3). The prime stability of the interlocking blocks allows to build the partition in columns (figure 4), so the wall can be built from fewer work stations, which means - shorter maneuvering time for the robot.

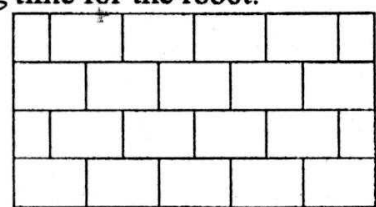

Figure 2: Building in stepped courses.

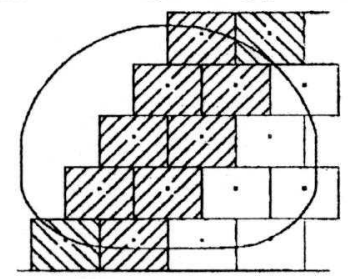

Figure 3: The reachable blocks in stepped courses.

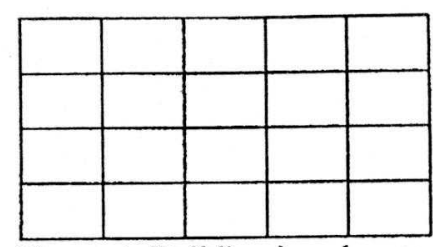

Figure 4: Building in columns.

The robot-integrated assembling process comprises of the following main sub-tasks: marking the position of the partition on the floor, gluing the partition tracks to the floor, marking the robot's work stations, driving the robot between the work stations, maneuvering the robot at the work station, stabilizing the robot in its work station, bringing the block pack to the work arca, positioning the block pack next to the robot, preparing the mortar, laying some blocks by humans, laying blocks by the robot, leveling the wall surface, filling the gaps among the blocks, gluing some sealing stripes, and transporting the robot through and between the floors. Some sub-tasks might be changed according to the features of the system.

The experiments that were conducted examined specific steps of the process of assembling a partition by blocks sized $65 \times 50 \times 9 \mathrm{~cm}$; each block weights nearly $30 \mathrm{~kg}$. The end-effctor was a dual-vacuum gripper, transforming common 8 atm. compressed-air pressure into vacuum. Since the shape of the work envelope resembles an ellipse, unlike the shape of the work sector, which is rectangular, there are some blocks that have to be laid by humans without the robot's help. It can be realized from figure 5 that the four comer blocks of the described work sector have to be laid by humans, as their gripping points (which define the TCP) are outside the work envelope.

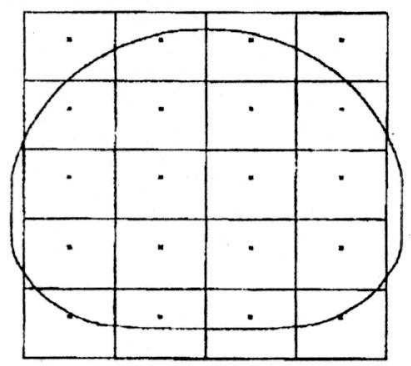

Figure 5: The reachable blocks in columns.

Each step in the asscmbling process was cxamined carefully, and its duration was either measured or estimated. Some of the estimates are based on experimental results, while others are bascd on observations at construction sites.

Three types of systems were examined. The usc of each type was determined with the aim of employing the robot as much as possible. 


\subsection{A robotic-assistant system}

A robotic assistant system is to help with the execution of the sub-tasks that are physically hard for humans, while the operator is to maintain the accuracy of the actions. In this system the autonomous robotic actions are very few and limited. Any transportation or maneuvering of the robot is done by the operator. The block laying stage is performed through close interaction between the operator and the robot. The robotic arm approaches the block pack to grip a block (figure 6), then the arm carries the block to the wall that is erccted and stops near its designated position. At this point the operator guides the arm using an appropriate Teach Pendant (TP), in order to lay the block precisely into its place (figure 7). While the robotic arm moves to get the next block, the operator spreads the mortar for it. The first cycle of any work station is somewhat more time consuming than the remaining cycles because the robot needs to "learn" where the block pack and the partition are.

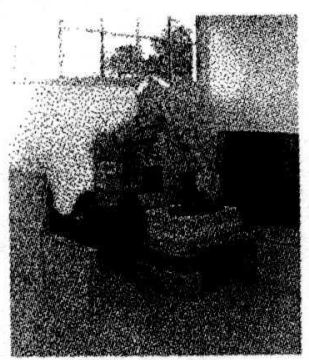

Figure 6:

Gripping a block.

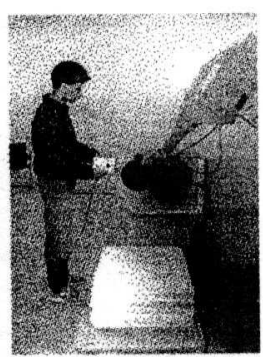

Figure 7:

Laying a block.
The analysis showed that the best work team that uses the robotic assistant system should be composed of an operator and a simple worker. In that way, the actions were categorized into three types:

1. Actions that employ both the robot and the operator.

2. Actions done by the operator alone. While executing these actions, the robot is unemployed (idle).

3. Actions done by the simple worker simultaneously with the working of the robot and/or the operator on other sub-tasks.

The detailed assignments were divided among the members of the work team in a table presented in figure 8. In that table, the sum of the first two columns (action type $1 \& 2$ ) determines the system's time input.

The following results were attained:

- The time input of the system is $5.03 \mathrm{~min} / \mathrm{sq} . \mathrm{m}$. ( 0.0839 hr/sq.m.).

- The part of each category out of total task duration: category no. $1=83 \%$; category no. $2=17 \%$; category no. $3=96 \%$.

- The robot is employed for $83 \%$ of the overall task processing time.

- The simple worker is employed for the whole task processing time.
- All of the assignments in category no. 2 have to be so because their execution obligates the robot to stay idle. Therefore, the suggested assignment division brings the system to maximum utiliz tion of the robot, and adding a second simple worker will not improve the performance of ths: work team.

\begin{tabular}{|c|c|c|c|}
\hline Sub-tasl: & $\begin{array}{c}\text { cat. } 1 \\
\text { robot \& } \\
\text { operator } \\
\text { [sec/w.st.] }\end{array}$ & $\begin{array}{l}\text { cat. } 2 \\
\text { operator } \\
\text { alone } \\
\text { [sec/w.st.] }\end{array}$ & $\begin{array}{c}\text { cat. } 3 \\
\text { simple } \\
\text { worker } \\
\text { [sec/w.st.] }\end{array}$ \\
\hline 1. & & & \\
\hline 2. & & & \\
\hline$:$ & & & \\
\hline $\mathrm{n}$. & & & \\
\hline Sum, [si cc/w.st.] & 1,633 & 330 & 1,877 \\
\hline $\begin{array}{l}\text { Sum } 0: \text { scrial } \\
\text { work [sic/w.st.] }\end{array}$ & \multicolumn{2}{|c|}{1,963} & \\
\hline $\begin{array}{l}\text { The part out of } \\
\text { task sturation } \\
{[\%]}\end{array}$ & $83 \%$ & $17 \%$ & $96 \%$ \\
\hline $\begin{array}{l}\text { The system's } \\
\text { time inf ut }\end{array}$ & \multicolumn{2}{|c|}{$0.0839 \mathrm{hr} / \mathrm{sq} . \mathrm{m}$. } & \\
\hline
\end{tabular}

w.st.=work station

Figure 8: Allocation of sub-tasks.

\subsection{A hi ghly autonomous robotic system}

is highly autonomous robotic system performs :almost the entire task autonomously (except certain su i-tasks, according to the system's features). The robct identifies its position relative to the surrounding, drives to a work station, identifies the block pack and begins the building process. Upon ending the work at the work station, the robot navigates and rides to the next work station. The operator is to supply the materials (blocks and mortar) a id inspect the robot's work. The operator also prerares the work area for the robot and compliments the sub-tasks that the robot couldn't do.

From the definition above it can be realized that the r rbotic system must sense the surrounding and evaluate it. A variety of devices should cnable the syster 1 to gauge its position, navigate and lay a block accurately. The system should be able to recognize the edges of a previous work sector in order to build the current one continuously. From the same reas on, the system should recognize the edges of the blicks that have just been laid. The system should also know the location of the gripping point of each blocis (that could be slightly different from one block to a nother).

A well known method for finding the orientation is based on triangulation, using a laser beam and (at least) three reflectors. This method can obtain gord results but has several disadvantages that make it less appropriate for the discussed system. Knowing the exact position of the reflectors relative 
to the laser origin is essential to the accuracy of the method, but these positions are hard to be determined while the reflectors and the robot are continuously transferred from one work area (e.g. room) to another. The method is also sensitive to the robot's inclination, that could differ from one work station to another. Moreover, even if this method is being used, still the accuracy of the manipulator movements is subjected to other errors that may occur, such as: deformations of the arm under different loads, errors in the location of the robot relative to its target, etc.

Realizing that an end-effetor must be, anyhow, designed to compensate for possible inaccuracies (such as those mentioned above), it is possible to use a less accurate orientation system. The analysis in this section assumes the utilization of a simple method for oricntation. In this method the operator must build the first row of blocks, and by that establish the exact layout of the partition. Then the operator sticks a few barcodes on that row in certain spots, to mark the work stations for the robot. The latter drives in parallel to the row of blocks, using a pair of distance sensors to keep a constant distance from the wall. A barcode reader mounted on the robot's platform searches for the barcode stickers, which may also include information about the work sector to be built. When a work station is recognized, the robot stops and begins the assembling process.

As a part of the assembling process, the end-effctor needs to identify the edges of the blocks that have already been laid, as well as the edge of the previous sector. Therefore, the operator should also build the first column of a partition. By this way, the robot can recognize horizontal and vertical guides for the exact location of the partition.

The analysis showed that the best work team that uses the autonomous system should be composed of an operator and a simple worker. In that way, the actions were categorized into five types:

1. Actions done autonomously by the robot.

2. Actions that employ both the robot and the operator together.

3. Actions done by the operator alone. While executing these actions, the robot is unemployed (idle).

4. Actions done by the operator simultaneously with the working of the robot on other sub-tasks.

5. Actions done by the simple worker simultaneously with the working of the robot and/or the operator on other sub-tasks.

The detailed assignments were divided among the members of the work team in a table presented in figure 9. In that table, the sum of the first threc columns (action type 1, 2 \& 3) determines the system's time input.

\begin{tabular}{|c|c|c|c|c|c|}
\hline & $\begin{array}{c}\text { cat. } \\
1\end{array}$ & $\begin{array}{c}\text { cat. } \\
2\end{array}$ & $\begin{array}{c}\text { cat. } \\
3\end{array}$ & $\begin{array}{c}\text { cat. } \\
4\end{array}$ & $\begin{array}{c}\text { cat. } \\
5\end{array}$ \\
\hline Sub-task & 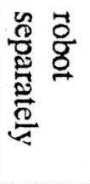 & 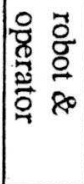 & 递营 & 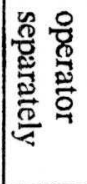 & 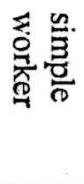 \\
\hline 1. & & & & & \\
\hline 2. & & I & & & \\
\hline$:$ & & & & & \\
\hline n. & & & & & \\
\hline Sum, [sec/w.st.] & 1,331 & 35 & 280 & 810 & 1,300 \\
\hline $\begin{array}{l}\text { Sum of serial } \\
\text { work [sec/w.st.] }\end{array}$ & \multicolumn{3}{|c|}{1,646} & & \\
\hline $\begin{array}{l}\text { The part out of } \\
\text { task duration } \\
{[\%]}\end{array}$ & $81 \%$ & $2 \%$ & $17 \%$ & $49 \%$ & $79 \%$ \\
\hline $\begin{array}{l}\text { The system's } \\
\text { time input }\end{array}$ & \multicolumn{3}{|c|}{$0.0704 \mathrm{hr} / \mathrm{sg} . \mathrm{m}$} & & \\
\hline
\end{tabular}

cat.=category

w.st. $=$ work station

Figure 9: Allocation of sub-tasks.

The following results were attained:

- The time input of the system is $4.22 \mathrm{~min} / \mathrm{sq} . \mathrm{m}$. (0.0704 hr/sq.m.).

- The part of each category out of total task duration: category no. $1=81 \%$; category no. $2=2 \%$; category no. $3=17 \%$; category no. $4=49 \%$; category no. $5=79 \%$.

- The robot is employed for $83 \%(=81+2)$ of the overall task processing time.

- The operator is employed for $68 \%(=49+17+2)$ of the overall task processing time.

- The actions done by the operator simultaneously with the robot actions are $49 \%$ of the overall task duration, while the duration of the autonomous robot-actions are 1.65 times longer. The duration of a continuous series of actions done by the robot autonomously in a work station is longer than the duration of the actions that need to be done by the operator. Therefore, the operator can reasonably manage to perform his actions.

- The simple worker is employed for $79 \%$ of the overall task processing time, so he has enough time to perform his job as well.

- All of the assignments in category no. 3 have to be so because their execution obligates the robot to stay idle. Therefore, the suggested assignment division brings the systcm to a maximum utilization of the robot, and adding a second simple worker will not improve the performance of the work team

\subsection{A semi-autonomous robotic system}

A semi-autonomous robotic system is a combination of the two systems discussed previously. Any transportation or mancuvering of the robot is done by the operator (like in a robotic assistant system), so that orientation systems and navigation abilities arc not necessary. The assembling stage is 
done autonomously by the robot (like in an autonomous system). The process of laying the blocks is based on using an end-effctor that identifies the already built parts of the wall. Thus, the operator still needs to build the first row and the first column of any partition.

The results that were attained show that the characteristics of the semi-autonomous robotic system are almost similar to those of the autonomous system.

\section{Economic analysis}

An cconomic analysis was conducted in order to examine and compare the profitability of the system types that were described in the previous section. The economic analysis is based on a method presented by Warszawski and Rosenfeld [13]. This method, with appropriate changes, allows to calculate the cost of producing one work unit, that is to say one sq.m. of partition.

The analysis takes into account several factors, as follows:

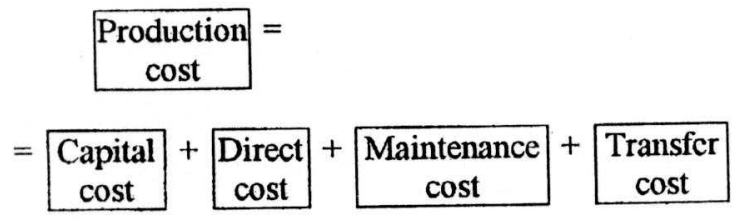

The analysis is based on the following assumptions:

1. The robotic system is multipurpose and can be employed for a total of 2,000 work hours per year.

2. The present common input of human work in building a gypsum block partition is 0.27 work hours/sq.m..

3. The annual interest ratc is $7 \%$.

4. The economic life span of the robot is 5 years.

5. The energy cost in operating the robot is $2 \$ / \mathrm{hr}$.

6. The annual repair cost (including labor, parts, and downtime) is $10 \%$ of the system's cost.

7. The routine maintenance is $6 \%$ of the cost of the working hours.

The transition from building by humans to working with either of the robotic systems requires not only buying the robot, but also employing another worker. This additional worker is the robot operator, and his wage may be higher than the wage of the simple worker who works beside hin.

\subsection{Results of the analysis}

Figurcs 10 and 11 present the profitable maximum cost of a robotic system dependent on the worker costs per hour.

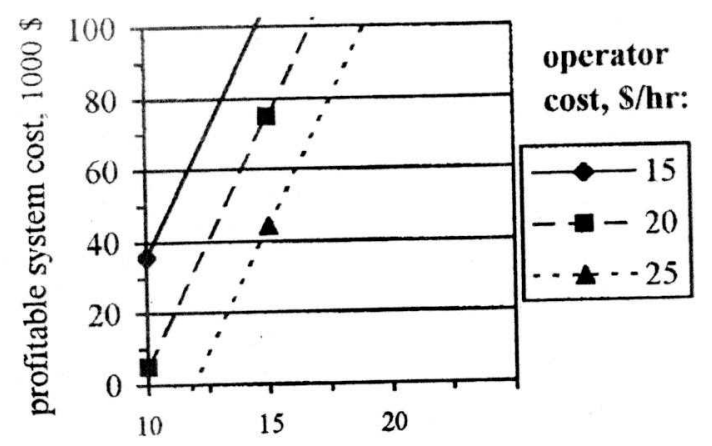

simple worker cost, $\$ / h r$

Figure 10: Profitability of transition from the present state to working with a robotic assistant system.

From figure 10 it can be scen, for example, that a robotic assistant system which will cost $60,000 \$$ will be profitable in the following circumstances:

- If a simple worker costs $14 \$ / \mathrm{hr}$ then the operator should cost no more than $20 \$ / \mathrm{hr}$.

- If a simple worker costs $12 \$ / \mathrm{hr}$ then the operator should cost no more than $15 \$ / \mathrm{hr}$.

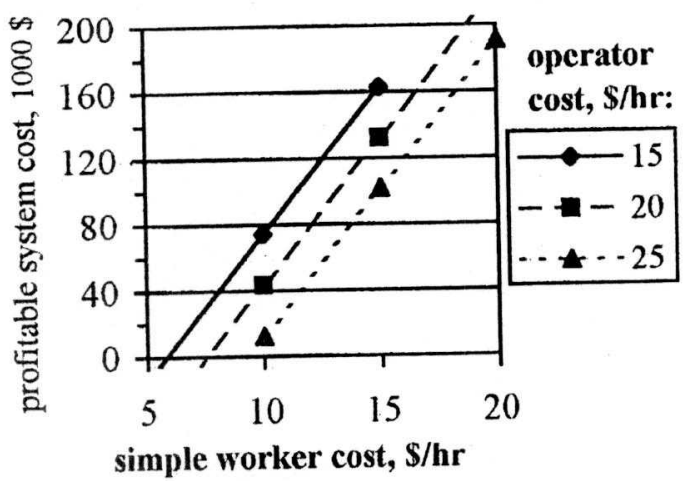

Figure 11: Profitability of transition from the present state to working with a semi-autonomous or autonomous system.

From figure 11 it can be secn, for cxample, that either a semi-autonomous or autonomous system which will cost $60,000 \$$ will be profitable in the following circumstances:

- If a simple worker costs $9 \$ / \mathrm{hr}$ then the operator should cost no more than $15 \$ / \mathrm{hr}$.

- If a simple worker costs $11 \$ / \mathrm{hr}$ then the operator should cost no more than $20 \$ / \mathrm{hr}$.

- If a simple worker costs $12.5 \$ / \mathrm{hr}$ then the operator should cost no more than $25 \$ / \mathrm{hr}$.

\section{Conclusions}

This paper examined three types of robotic systems for partition construction. These systems differ from each other by their level of autonomy and by the task-allocation between the humans and the robot. Each type was determined in the best possible way for its category and an cconomic analysis was conducted. 
The following conclusions can be drawn:

1. Working with a robotic assistant system reduces the task duration by $70 \%$. Working with a semi-autonomous or autonomous system reduces the task duration by $75 \%$.

2. The use of large blocks utilizes the robot's advantage of high physical strength and, at the same time, it contributes to lowering of the total time input.

3. Assigning more sub-tasks to the simple worker, who works with the robotic assistant system, will affect the system's performance, because this worker is fully occupied. Therefore, this system will be better used in a well-planned modular structure, where less complementary sub-tasks are to be done by the simple worker. However, the simple worker who works with the semi-autonomous or the autonomous system is free to get some more sub-tasks without affecting the system's efficiency.

4. The semi-autonomous and the autonomous systems (as described in this paper) achieve similar performance, so, in the short term, it is not beneficial to invest in developing expensive positioning systems.

5. The wage level in a certain construction market is a crucial factor in determining the profitability of a robotic system. The relation between the profitability and the wage level is visually presented and conclusions can be drawn according to the local circumstances.

\section{References}

1. Rosenfeld Y., Warszawski A and Zajicek U., "Full Scale Building with Interior Finishing Robot", International Journal of Automation in Construction, Vol. 2, No. 4, 1993, pp. 229-240.

2. Warzhawski A. and Rosenfeld Y., "Robot for Interior Finishing Works in Building - Feasibility Analysis", Journal of Construction Engineering and Management, ASCE (American Society of Civil Engineers), Vol. 120, No. 1,1994, pp. 132-151.

3. "Rosenfeld Y., Shohet I.M. and Warszawski A., "Optimization Algorithms of Task-Planning for Construction Robots", International Journal of Costruction Information Technology, Vol. 2, No. 3, 1994, pp. 1-19.

4. Andres J., Bock T., Gebhart F. and Steck W., "First Results of Development of the Masonry System ROCCO: A Fault Tolerant Assembly Tool", Proceedings of The 11th International Symposium on Automation and Robotics in Construction (ISARC), 1994, Brighton, U.K., pp. 87-93.

5. Pritschow G., Dalacker M., Kurz J. and Gaenssle M., "Technological Aspects in the Development of a Mobile Bricklaying Robot", Proceedings of The 12th International Symposium on Automation and Robotics in Construction (ISARC), 1995, Warzawa, Poland, pp. 281-290.

6. Balaguer C., Gambao E., Barrientos A., Puente E.A. and Aracil R., "Site Assembly in Costruction Industry by Means of A Large Advaced Robot", Proceedings of the 13th International Symposium on Automation and Robotics in Construction (ISARC), 1996, Tokyo, Japan, pp. 65-72.

7. Kloek R., Bos J. and Marck R., "The Development and Testing of a Mobile Drilling Robot", Proceedings of the 11th International Symposium on Automation and Robotics in Construction (ISARC), 1994, Brighton, U.K., pp. 63-70.

8. Tanaka k., Kajitani M., Kanamori C., Itoh H., Abe Y. and Tanaka Y., "Development of Marking Robot Working at Building Sites", Procecdings of the 12th International Symposium on Automation and Robotics in Construction (ISARC), 1995, Warzawa, Poland, pp. 235-242.

9. Pritschow G., Kurz J., McComac S.E. and Dalacker M., "Practical Sensor Strategies for On-Site Positioning of a Mobile Bricklaying Robot", Proceedings of the 13th International Symposium on Automation and Robotics in Construction (ISARC), 1996, Tokyo, Japan, pp. 895-904.

10. Miyama A., Sawada M. and Suzuki I., "Development of a Multifunctional Robot for Installation of Building Exterior and Interior Finishing Materials", Proceedings of the 11th International Symposium on Automation and Robotics in Construction (ISARC), 1994, Brighton, U.K., pp. 709-716.

11. Yoshinada H., Sawano T., Takahoshi I., Suzuki H., Muro E. and Hosino H., "Development of Board Placing Robot", Proceedings of the 13th International Symposium on Automation and Robotics in Construction (ISARC), 1996, Tokyo, Japan, pp. 877-886.

12. Nanba K., Takasu M., Sato T. and Kojima S., "Development and Application of the Light Weight Manipulator for Interior Finish Work", Proceedings of the 13th International Symposium on Automation and Robotics in Construction (ISARC), 1996, Tokyo, Japan, pp. 887-894.

13. Warszawski A. and Rosenfeld Y., "Economic Analysis of Robots Employment in Building", Proceedings of the 14th International Symposium on Automation and Robotics in Construction (ISARC), 1997, Pittsburgh, Pennsylvania, U.S.A., pp. 177-184 